Check for updates

Cite this: Phys. Chem. Chem. Phys., 2021, 23, 26349

Received 30th September 2021 Accepted 12th November 2021 DOI: $10.1039 / d 1 c p 04483 d$

rsc.li/pccp

\section{Exotic nuclear spin behavior in dendritic macromolecules $\dagger$}

\author{
Philip Saul, (D) ${ }^{\text {ab }}$ Shengjun Yang, $\ddagger^{\mathrm{ab}}$ Salvatore Mamone, ${ }^{\mathrm{ab}}$ Felipe Opazo, (D) $^{\mathrm{bc}}$ \\ Andreas Meyer, ${ }^{d}$ Silvio O. Rizzolic and Stefan Glöggler (D)*ab
}

Dendrimers are a class of branched, highly symmetric macromolecules that have been shown to be useful for a vast number of different applications. Potential uses as fluorescence sensors, in catalysis and perhaps most importantly in medical applications as drug delivery systems or cytotoxica have been proposed. Herein we report on an exotic behaviour of the nuclear spins in a dendritic macromolecule in the presence of different paramagnetic ions. We show that the stability of the long lived nuclear singlet state, is affected by the presence of $\mathrm{Cu}(I)$, whereas other ions did not have any influence at all. This effect could not be observed in the case of a simple tripeptide, in which the nuclear singlet stability was influenced by all investigated paramagnetic ions, a potentially useful effect in the development of $\mathrm{Cu}(॥)$ selective probes. By adding a fluorescent marker to our molecule we could show that the nuclear singlet multimer (NUSIMER) is taken up by living cells. Furthermore we were able to show that nuclear singlet state NMR can be used to investigate the NUSIMER in the presence of living cells, showing that an application in in vivo NMR can be feasible.

\section{Introduction}

Dendrimers are large, centrosymmetric, highly branched macromolecules that can be easily modified at the end of each branch by different means of chemical synthesis. ${ }^{1}$ Since the first report on dendritic structures, ${ }^{2}$ dendrimers quickly raised the interest of researchers in different fields and a large number of different possible applications have been proposed, ranging from the use as effective filtering systems ${ }^{3}$ over the use as chemical sensors ${ }^{4,5}$ to chemical catalysis. ${ }^{6,7}$ In the latter two cases, the high number of terminal functional groups in dendrimers drastically increases the effectiveness in the respective applications, since they allow for a large number of active residues to be condensed in one molecule and thus in the same area. Apart from the aforementioned possibilities, dendrimers

\footnotetext{
${ }^{a}$ Research Group for NMR Signal Enhancement, Max Planck Institute for Biophysical Chemistry, Am Fassberg 11, 37077 Göttingen, Germany.

E-mail: stefan.gloeggler@mpibpc.mpg.de; Tel: +49 5513961108

${ }^{b}$ Center for Biostructural Imaging of Neurodegeneration, Von-Siebold-Straße 3A, 37075 Göttingen, Germany

'Institute for Neuro- and Sensory Physiology, University Medical Center Göttingen, Humboldtallee 23, 37073 Göttingen, Germany

${ }^{d}$ Research Group Electron Paramagnetic Resonance, Max Planck Institute for Biophysical Chemistry, Am Fassberg 11, 37077 Göttingen, Germany

$\dagger$ Electronic supplementary information (ESI) available. See DOI: 10.1039/d1cp04483d \$ Present address: Key Laboratory of Pesticide and Chemical Biology of Ministry of Education, Hubei International Scientific and Technological Cooperation Base of Pesticide and Green Synthesis, College of Chemistry, Central China Normal University, Wuhan, 430079, China.
}

have been investigated especially in the field of medical research. Cavities in the dendritic structures allow for the use as drug delivery systems by encapsulating different compounds. ${ }^{8,9}$ Additionally, dendrimer based cytotoxica have been introduced by attaching small cytotoxic compounds to dendrimers, again accumulating a high number of active compounds in the same place. ${ }^{10-12}$

A fairly recent proposal for the use of dendrimers are nuclear singlet multimers (NUSIMERs). ${ }^{13}$ This approach makes use of the accumulation of up to 128 equivalent residues at the branches of a poly(amidoamide) dendrimer of the 5th generation (G5-PAMAM) in combination with nuclear singlet state NMR. A nuclear spin singlet state requires two spin-12 nuclei coupling with each other. While the triplet state with a total spin of 1 , can be observed in regular NMR experiments, the singlet state with a total spin of 0 is NMR silent. ${ }^{14}$ Nuclear singlet states have been shown to have lifetimes often exceeding the spin-lattice relaxation $T_{1}$. This particular property allows for the transfer of magnetization from the triplet state to the singlet state, in which the spin order can be stored and, at a later point, retrieved again. ${ }^{14-44} \mathrm{~A}$ combination of the transfer of magnetization to singlet state order and back with a singlet filter allows for selective observation of just the proton pair in which a singlet state has been populated, thus eliminating all background signal, including the solvent signal. ${ }^{13,44,45}$ For a better understanding of the behaviour of the nuclear spins, this phenomenon has been thoroughly investigated in perfect environments, such as low viscosity, perdeuterated 
solvents ${ }^{34}$ or under the exclusion of oxygen in order to minimize singlet leakage and thus extending the singlet state lifetime. ${ }^{15,21,33}$ The fact that the equilibration time between the NMR silent nuclear singlet state and the NMR active triplet state $\left(T_{\mathrm{S}}\right)$ oftentimes exceeds $T_{1}{ }^{20-23}$ leads to the possibility to analyze dynamics and has already been used to probe structures of proteins and to investigate drug binding as well as diffusion phenomena ${ }^{41-44,46,47}$ or even the selective detection of different amino acids in the same protein in vitro. ${ }^{48}$ However, most of those experiments have been carried out in deuterated solvents, degassed samples or with partially deuterated compounds as well. In few studies it could be shown that long-lived nuclear singlet states can be used in vitro in non-deuterated solvents and in near physiological conditions, ${ }^{13,44}$ or even in vivo in magnetic resonance spectroscopy to filter metabolic signals. ${ }^{49}$ In the latter example it could be shown that singlet filtering is possible in MRI experiments. Especially in this context, the inherently low sensitivity of NMR can be somewhat overcome by the accumulation of a large number of chemically equivalent proton pairs in the same molecule, for which the use of dendrimers is exceptionally well suited. The fact that dendritic structures have been shown to be a valid concept in the development of probes, lead us to investigate the possibilities to use dendritic structures to probe for paramagnetic ions and furthermore explore possibilities to use NUSIMERs in in-cell experiments.

\section{General concept}

A nuclear singlet multimer (NUSIMER) G5-PAMAM-GGA-NH $\mathrm{N}_{2}$, is a poly(amidoamide) dendrimer of the 5th generation (G5-PAMAM) which has been modified by attaching the glycin-glycin-alanin tripeptide (GGA) (1) to it to obtain a macromolecule (2) that contains up to 128 proton pairs per molecule with the same chemical shift dispersion in which a nuclear singlet state can be populated ${ }^{13}$ (Fig. 1). Due to the high density of chemically equivalent proton pairs in which a singlet state can be populated, the use of NUSIMERs can greatly increase the sensitivity and NUSIMERs can be detected at low concentrations in reasonably quick experiments. The presence of amide and amine functions within the structure as well as at the end of each branch, makes them excellent candidates for the interaction with metal ions and thus the potential use as probes for metal ions.

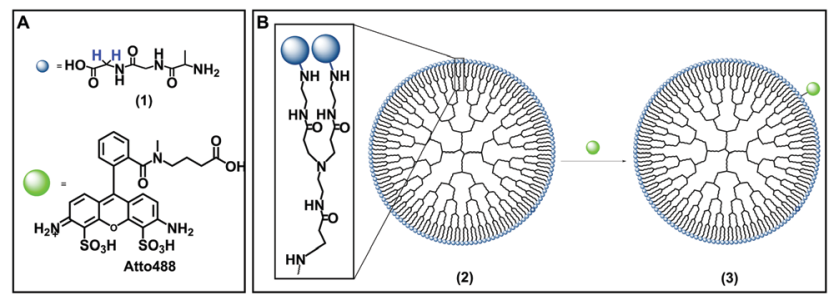

Fig. 1 (A) Structure of the tripeptide (GGA) (1) with accessible proton singlet states indicated in blue and structure of the fluorescent tag Atto488. (B) Modification of G5-PAMAM-GGA-NH $\mathrm{N}_{2}$ (2) by addition of Atto488 leads to G5-PAMAM-GGA-Atto488 (3).
In a first set of experiments we investigated the effect of paramagnetic metal ions on $T_{1}^{-1}$ and $T_{\mathrm{s}}{ }^{-1}$ in (1) and (2) to assess the possibility to use NUSIMERs to sense certain ions. In an investigation of the effect of some paramagnetic ions on $T_{1}$ and $T_{\mathrm{s}}$ in a simple glycine-alanine dipeptide, it has been shown that the singlet state is less sensitive to paramagnetic relaxation than the conventional nuclear magnetization. ${ }^{40}$ This property can be useful in increasing the specificity compared to the standard NMR experiment. Metal ions play a pivotal role in many biological processes. Especially $\mathrm{Cu}(\mathrm{II})$ has gained some attention, since high $\mathrm{Cu}(\mathrm{II})$ concentrations are associated with the formation of $A \beta$-plaques which can be found in Alzheimer's disease patients. ${ }^{50}$ Furthermore, we investigated the effect of Fe(III), Gd(III), Mn(II) and $\mathrm{Zn(II)} \mathrm{on} T_{1}^{-1}$ and $T_{\mathrm{s}}{ }^{-1}$.

To further explore the possibility to use NUSIMERs for the detection of said ions in a biological environment, we decided to investigate, if NUSIMERs can be viable for in vivo experiments in cells. In order to be able to trace the NUSIMER, compound (2) has been modified by attaching a fluorescent tag (Atto488) to it to obtain G5-PAMAM-GGA-Atto488 (3) (Fig. 1).

\section{Results and discussion}

Influence of paramagnetic metal ions on $T_{1}{ }^{-1}$ and $T_{\mathrm{s}}{ }^{-1}$

In order to simulate the environment most likely to be relevant in potential in vivo applications, samples of (2) have been prepared in aqueous HEPES buffered solutions (10 mM) and have been titrated with $\mathrm{Gd}(\mathrm{III}), \mathrm{Mn}$ (II), $\mathrm{Fe}(\mathrm{III}), \mathrm{Cu}(\mathrm{II})$ and $\mathrm{Zn}$ (II) respectively (experiments conducted at $600 \mathrm{MHz}$ ). $\mathrm{Zn}$ (II) is diamagnetic but also an important disease marker and was hence included in our investigation. After each addition of the respective ions $T_{1}^{-1}$ and $T_{\mathrm{s}}{ }^{-1}$ have been determined $\left(T_{1}^{-1}\right.$ plots are provided in the ESI $\dagger$ ). We performed the same experiments with (1) to correlate the obtained data (Fig. 2).

For (1) we observed changes in $T_{1}^{-1}$ and $T_{\mathrm{s}}{ }^{-1}$ in a similar relation in the presence of paramagnetic ions as has been shown before. ${ }^{40}$ Notably, in the case of (1) in combination with Fe(III) we observed an effect on $T_{1}^{-1}$ and $T_{\mathrm{s}}{ }^{-1}$ which can be explained by the slow complexation of Fe(III) by the HEPES buffer. The complexation of Fe(III) by different Goods buffers has been reported previously. ${ }^{51}$ We investigated this complexation more thoroughly by determining $T_{1}$ and $T_{\mathrm{s}}$ in a sample of (1) in HEPES buffer at different times after the addition of $\mathrm{Fe}$ (III) $(150 \mu \mathrm{M})$. We could observe a slow increase in $T_{1}$ and $T_{\mathrm{s}}$ respectively (Fig. 2f). To show that this is an effect of the buffer as well as exclude any effect of the buffer in all cases, we investigated the observed phenomena in unbuffered $\mathrm{D}_{2} \mathrm{O}$ solutions of the paramagnetic ions, whereby the $\mathrm{pD}$ remained unchanged and independent of the ion concentration. These investigations have been conducted at a lower magnetic field $(300 \mathrm{MHz}$ ) to exclude field dependency effects. We found that $T_{1}{ }^{-1}$ and $T_{\mathrm{s}}^{-1}$ of the peptides and dendrimer investigated display a similar behavior as in the buffer, with $\mathrm{Fe}(\mathrm{III})$ following the expected behavior as it does not form a complex (Fig. 3a). In case of (2) we could not observe any effect on either $T_{\mathrm{s}}{ }^{-1}$ or $T_{1}{ }^{-1}$ 

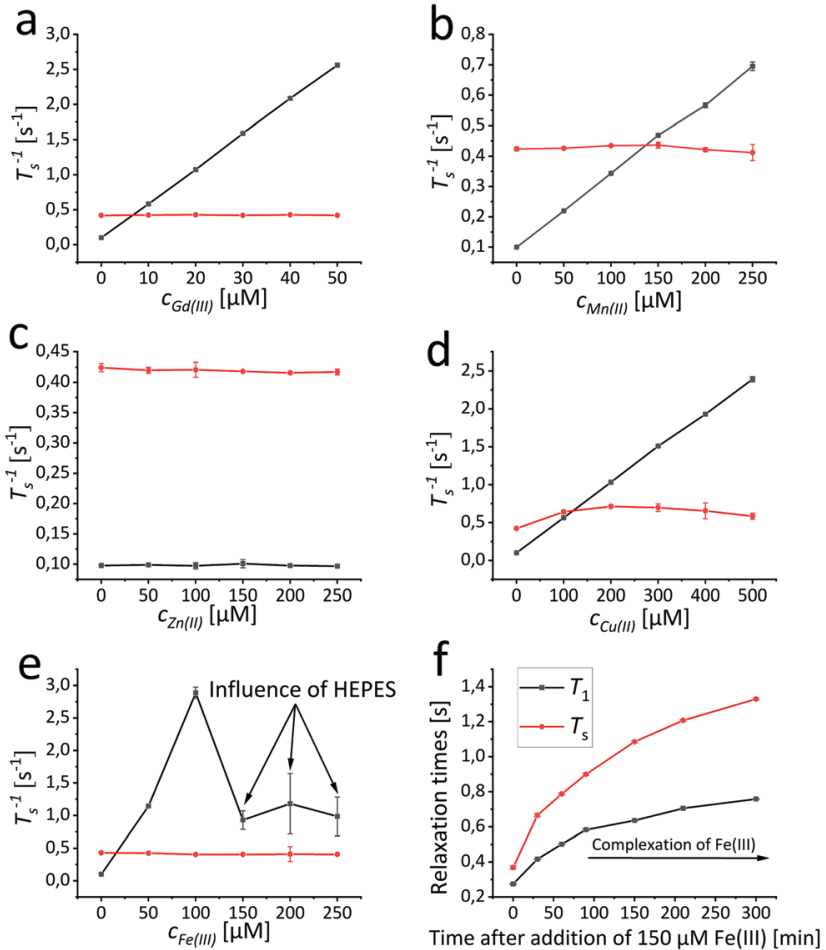

Fig. 2 Equilibration rates $T_{\mathrm{s}}^{-1}$ of GGA (12.8 mM) (1) (black) and G5PAMAM-GGA-NH $\mathrm{N}_{2}\left(100 \mu\right.$ M) (2) (red) in HEPES buffered $\mathrm{H}_{2} \mathrm{O}$ at $600 \mathrm{MHz}$ depending on the concentration of different paramagnetic ions $\mathrm{Gd}($ (II) (a), $\mathrm{Mn}$ (II) (b), $\mathrm{Zn}$ (II) (c), $\mathrm{Cu}\left(\right.$ II) (d) and $\mathrm{Fe}\left(\right.$ (II) (e). An effect of $\mathrm{Cu}\left(\right.$ II) on $T_{\mathrm{s}}$ in the dendrimer is apparent in (d). This effect cannot be seen for the other ions within the error. The effect of Fe(III) on $T_{\mathrm{s}}$ in the tripeptide (e) can be explained by the interaction of HEPES with Fe(III) (f). Error margins are standard deviations of 3 separate measurements with 8 scans each. a
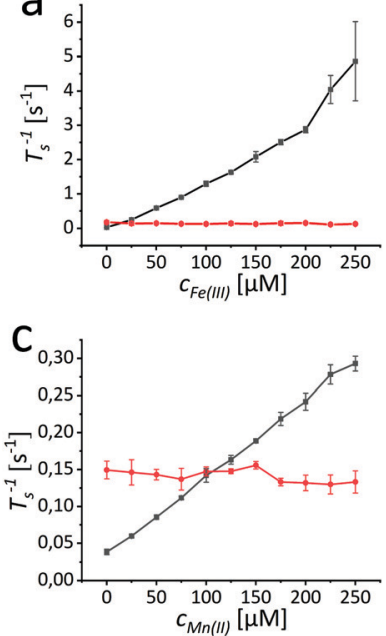

$b_{1,6}$
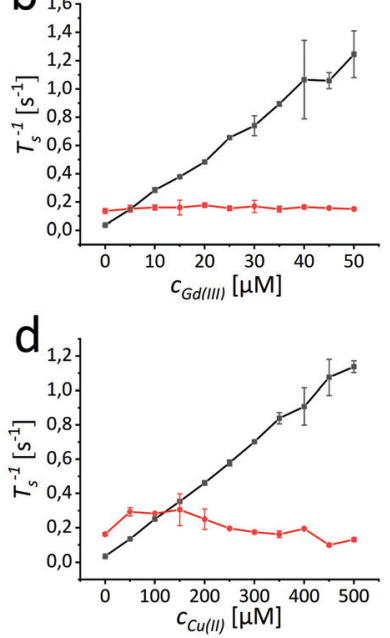

Fig. 3 Equilibration rates $T_{\mathrm{s}}^{-1}$ of (1) $(12.8 \mathrm{mM})$ (black) and (2) $(100 \mu \mathrm{M})$ (red) in $\mathrm{D}_{2} \mathrm{O}$ at $300 \mathrm{MHz}$ depending on different concentrations of $\mathrm{Fe}(\mathrm{III})$ (a), $\mathrm{Gd}(\mathrm{II})$ (b), $\mathrm{Mn}(॥)$ (c) and $\mathrm{Cu}(॥)$ (d). Error margins are the standard deviation of 3 different measurements.

except in the case of $\mathrm{Cu}$ (II) (Fig. 2d). This effect of $\mathrm{Cu}$ (II) is especially apparent at concentrations between $200 \mu \mathrm{M}$ and
$400 \mu \mathrm{M}$, which are the concentrations reported to be found in $\mathrm{A} \beta$ aggregates as well. ${ }^{50}$ If one closely examines the effect of copper on $T_{\mathrm{s}}^{-1}$, an increase in the singlet stability rate is experienced at first which reaches a minimum at $150 \mu \mathrm{M}$ copper concentration. If the copper concentration is further increased, $T_{\mathrm{s}}{ }^{-1}$ decreases as well. For copper we investigated if we can exclude effects of the buffer or if we, similarily to iron, see an interaction of buffer with the ions. Upon addition of copper to the dendrimer we observe in $\mathrm{D}_{2} \mathrm{O}$ that $T_{\mathrm{s}}{ }^{-1}$ starts subceeding the initial value found in the dendrimer, when no ions had been added. We did not observe this behavior if $T_{1}{ }^{-1}$ is investigated. $T_{1}{ }^{-1}$ increases with copper concentration and reaches a plateau and did not go down again within the error over the investigated range.

We tried to confirm this behaviour at a higher magnetic field and observed a similar effect in $\mathrm{D}_{2} \mathrm{O}$ at $600 \mathrm{MHz}$, although $T_{\mathrm{s}}{ }^{-1}$ did not subceed the initial value. The same effect could be observed for $T_{1}^{-1}$ (Fig. 4).

For an explanation of the effect of $\mathrm{Cu}(\mathrm{II})$ on $T_{\mathrm{s}}{ }^{-1}$ we propose a direct influence of the ions on the constitution of (2). In dendrimers, typically three possible mechanisms are considered how ions can influence the constitution. They can interact with the chains, be incorporated into the center of the dendrimer and may be influenced by electrostatic repulsion of charged parts of the dendrimer and ions. ${ }^{52-55}$ Once copper is added, we observe complexation of $\mathrm{Cu}(\mathrm{II})$ to the amide functions at first. This interaction of $\mathrm{Cu}(\mathrm{II})$ with the amide functions is confirmed in UV/vis experiments (see ESI $\dagger$ ). At a certain copper concentration, a saturation of the amide functions at the outer sphere seems to occur. With a further increase in copper concentration we hypothesize that the chain mobility further increases whereby no further copper ions have an effect on the singlet state equilibration. As a result, $T_{\mathrm{s}}{ }^{-1}$ would become shorter due to an ongoing decrease in correlation time. A similar behaviour has been described for the interaction of the inner branches of a poly(amidoamide) dendrimer of the 4 th generation with $\mathrm{Cu}(\mathrm{II}){ }^{56}$

In order to verify this hypothesis, we performed diffusionordered spectroscopy (DOSY) measurements to analyze the change of chain mobility at increasing copper concentrations and how $T_{1}^{-1}$ and $T_{\mathrm{s}}{ }^{-1}$ are influenced. A first result that we found is that the diffusion coefficient $(D)$ of the glycine protons in the dendrimer increases linearly with the copper

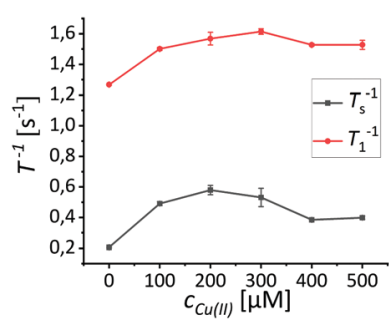

Fig. 4 Equilibration rates $T_{\mathrm{s}}^{-1}$ (black) and $T_{1}^{-1}$ (red) of (2) $(100 \mu \mathrm{M})$ in $\mathrm{D}_{2} \mathrm{O}$ depending on different concentrations of $\mathrm{Cu}(\mathrm{II})$ at $600 \mathrm{MHz}$. Error margins are the standard deviation of 3 different measurements. 
a
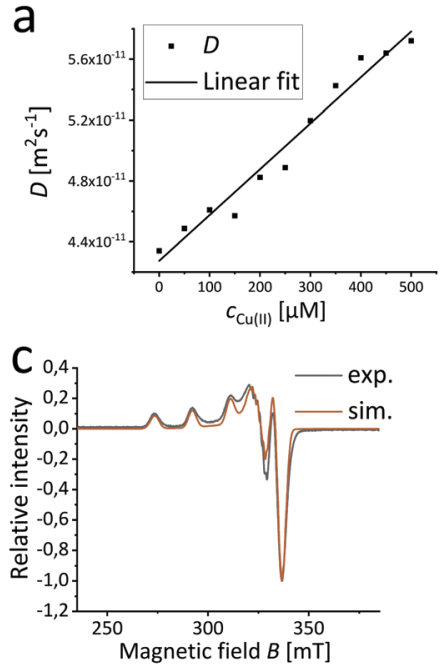

Fig. 5 (a) Diffusion coefficient (D) at different $\mathrm{Cu}(\Perp)$ concentrations as determined via DOSY NMR spectroscopy. The effect on the diffusion coefficient is $3 \times 10^{-14} \mathrm{~m}^{2} \mathrm{~s}^{-1}$. (b) EPR spectra of $\mathrm{Cu}(\mathrm{II})(500 \mu \mathrm{M})$ in the presence of GGA (black) and $\mathrm{Cu}(\|)(500 \mu \mathrm{M})$. (c) EPR spectrum of $\mathrm{Cu}(\mathrm{II})$ $(500 \mu \mathrm{M})$ in the presence of G5-PAMAM (black) and simulated spectrum (red). (d) EPR spectrum of $\mathrm{Cu}(\mathrm{II})(500 \mu \mathrm{M})$ in the presence of (2) (black) and simulated spectrum (red). The spectra have been recorded at $30 \mathrm{~K}$ in a mixture of $\mathrm{D}_{2} \mathrm{O}$ and glycerol $(1: 2)$.

concentration (Fig. 5a). At the same time $T_{\mathrm{s}}^{-1}$ in the dendrimers increases sharply and then starts to decrease again. This effect, can be observed at $150 \mu \mathrm{M} \mathrm{Cu}$ (II) in $T_{\mathrm{s}}^{-1}$ whereby a plateau is reached for $T_{1}^{-1}$ at similar concentrations within the error. The increase in $\mathrm{Cu}$ (II) concentration did not show any effect on the diffusion coefficient of the water signal (data shown in the ESI $\dagger$ ), showing that no change in viscosity takes place. Thus a contribution of a possible viscosity change can be excluded, as can be an effect of the buffer or the solvent, due to the effect not being observed in the case of the tripeptide GGA in buffered and unbuffered solutions (see ESI $\dagger$ ).

To further investigate the kind of interaction between $\mathrm{Cu}(\mathrm{II})$ and (2), we recorded electron paramagnetic resonance (EPR) spectra of $\mathrm{Cu}(\mathrm{II})(500 \mu \mathrm{M}), \mathrm{GGA}$ in the presence of $\mathrm{Cu}(\mathrm{II})$ $(500 \mu \mathrm{M})$, G5-PAMAM with $500 \mu \mathrm{M} \mathrm{Cu}(\mathrm{II})$ as well as (2) with $500 \mu \mathrm{M} \mathrm{Cu}(\mathrm{II})$ (Fig. 5). Additionally, simulations of the experimental spectra have been performed.

In a first experiment the EPR spectrum of $\mathrm{Cu}$ (II), or rather hexaaqua- $\mathrm{d}_{12} \mathrm{Cu}(\mathrm{II})$, in $\mathrm{D}_{2} \mathrm{O}$ was recorded as a reference spectrum. This spectrum showed to be identical to the obtained spectrum of $\mathrm{Cu}(\mathrm{II})$ in $\mathrm{D}_{2} \mathrm{O}$ in the presence of GGA, indicating that no complexation of $\mathrm{Cu}(\mathrm{II})$ by GGA takes place (Fig. $5 \mathrm{~b}$ ). This observation has also been confirmed by UV/VIS measurements (see ESI $\dagger$ ). The measurements for $\mathrm{Cu}(\mathrm{II})$ in the presence of G5PAMAM and (2) (Fig. 5c and d) display different spectra not only to the one of hexaaqua $\mathrm{Cu}(\mathrm{II})$, with a $g_{z}$ of 2.4 , but also to one another. Performed simulations indicated a ligation by the nitrogen atoms of the dendrimer and the $\mathrm{Cu}(\mathrm{II})$ with a $g_{z}$ at around 2.25 which is significantly different than the one of the hexaaqua complex of $\mathrm{Cu}$ (II) and characteristic for $\mathrm{Cu}(\mathrm{II})-\mathrm{N}$ complexes. ${ }^{57-60}$ In both cases the shape of the EPR spectra were independent of the $\mathrm{Cu}$ (II) concentration. The simulations point towards a tetragonal symmetry around the $\mathrm{Cu}(\mathrm{II})$ in the presence of G5-PAMAM, with equal $g_{x}$ and $g_{y}$, whereas in the presence of (2) a rhombic symmetry is obtained from the simulation where $g_{x}, g_{y}$ and $g_{z}$ are all different from one another. It is, however, likely that the experimental spectra contain contributions from different $\mathrm{Cu}(\mathrm{II})$ complexes as has been shown to be the case in the presence of G4-PAMAM. ${ }^{56}$ The parameters obtained from the simulations are shown in the ESI. $†$

We herein propose a model in which, upon the first addition of $\mathrm{Cu}$ (II) to the dendrimer, a coordination of $\mathrm{Cu}(\mathrm{II})$ to the terminal amino functions as well as a coordination to the amide functions of the tripeptide residues occurs. This leads to an increase in the diffusion coefficient of the glycine residues and thus to an increased chain mobility. However upon further addition of $\mathrm{Cu}$ (II) the chain mobility of the tripeptide residues further increases and a decrease in $T_{\mathrm{s}}{ }^{-1}$ takes place. The first increase in $T_{\mathrm{s}}^{-1}$ is likely induced by the binding of copper to the amide functions of the tripeptide resdiues and the thus increased proximity to the glycine protons. The subsequent decrease in $T_{\mathrm{s}}{ }^{-1}$ then can be attributed to the further increasing mobility of the protons. The decrease in $T_{\mathrm{s}}{ }^{-1}$ is likely due to a difference in the distance dependency of $T_{1}{ }^{-1}$ and $T_{\mathrm{S}}^{-1}$ of the dipole-dipole relaxation $\left(r_{6} \text { for } T_{1}^{-1} \text { and } r_{8} \text { for } T_{\mathrm{s}}{ }^{-1}\right)^{61}$ which might indicate a saturation of the outer sphere of the dendrimer with $\mathrm{Cu}(\mathrm{II})$ which then does only allow further $\mathrm{Cu}(\mathrm{II})$ to have an effect on $T_{1}{ }^{-1}$ since it cannot get any closer to the relevant protons. Any further $\mathrm{Cu}$ (II) is likely incorporated into the dendritic core, an effect that has been made use of e.g. in dendrimer entrapped nanoparticles. ${ }^{52,62}$

At the point of the incorporation of $\mathrm{Cu}$ (II) into the dendritic core, a decrease in hydrodynamic radius of the dendrimers is likely to occur. Due to the difference in chain mobility a direct correlation of the diffusion coefficient determined by DOSY NMR and the hydrodynamic radius is challenging. ${ }^{63}$ For further insight into a possible change in size of the dendrimers, dynamic light scattering (DLS) measurements have been performed (see ESI $\dagger$ ). The size of G5-PAMAM-GGA was thus determined to be around $3.5 \mathrm{~nm}$ which is in the order of magnitude of the determined size of G5-PAMAM. ${ }^{55}$ However no significant change in particle size upon the addition of $\mathrm{Cu}(\mathrm{II})$ could be determined. This indicates that $\mathrm{Cu}$ (II) at low concentrations, mainly interacts with the outer branches of the dendrimer, influencing their mobility, while the impact on the particle size seems to be minimal, if at all present.

The binding hypothesis is supported by the observation of a change in color upon the addition of (2) to a solution of $\mathrm{Cu}(\mathrm{II}) \mathrm{Cl}_{2}$ from a nearly colorless light blue to a considerably darker blue (see Fig. S24 in the ESI $\dagger$ ). As dendrimers represent a complex system, the proposed mechanism above may serve as another step to support ongoing work on solving the structures of dendritic molecules. As already described above, this change is observed by UV/vis spectroscopy showing a change in absorption bands typically associated with amide bonds in proteins 
upon addition of $\mathrm{Cu}$ (II) to a solution of (2). We did not observe this change in the case of Gd(III), Mn(II) and Fe(III) (See Fig. S12S23 in the ESI $\dagger$ ).

\section{Assessment of NUSIMERs as potential bioprobes}

The observed selective effect of $\mathrm{Cu}(\mathrm{II})$ on $T_{1}{ }^{-1}$ and $T_{\mathrm{s}}{ }^{-1}$ gives rise to a possible application of the NUSIMERs as $\mathrm{Cu}(\mathrm{II})$ probes, especially, but not limited to, the detection of $\mathrm{Cu}(\mathrm{II})$ in cells or tissue. This is especially interesting due to the central role of $\mathrm{Cu}$ (II) in the formation of $\mathrm{A} \beta$ plaques. We would like to note that changes in $T_{\mathrm{s}}$ are already sensed below $100 \mu \mathrm{M}$ copper concentrations which is well in the region in what is found in extracellular $A \beta$ plaques. In order to test the viability of future tissue experiments, we performed additional measurements of a solution of (2) in an agarose gel which simulates the consistency of brain matter. ${ }^{64}$ In this case, two experiments have been performed: In both cases, the dendrimers were measured at a concentration of $10 \mu \mathrm{M}$ in gel which consisted of a HEPES (4-(2-hydroxyethyl)-1-piperazineethanesulfonic acid) buffered solution. One of the samples additionally contained $50 \mu \mathrm{M}$ of $\mathrm{Cu}$ (II). Relaxation rate experiments gave a $T_{1}^{-1}$ of $0.016 \mathrm{~s}^{-1}$ and a $T_{\mathrm{s}}^{-1}$ of $2.42 \pm 0.06 \mathrm{~s}$ for the sample without $\mathrm{Cu}(\mathrm{II})$ and $T_{1}{ }^{-1}$ of $2.32 \mathrm{~s}^{-1}$ and a $T_{\mathrm{s}}^{-1}$ of $0.69 \mathrm{~s}^{-1}$ for the sample with $\mathrm{Cu}$ (II). These findings indicate that the same effect thoroughly studied in $100 \mu \mathrm{M}$ concentrations in $\mathrm{D}_{2} \mathrm{O}$ can be observed in lower concentrations in a high viscosity environment and in the presence of buffered $\mathrm{H}_{2} \mathrm{O}$ solutions. $T_{\mathrm{s}}{ }^{-1}$ appears thereby to be less sensitive to the presence of copper than $T_{1}^{-1}$. Those findings strongly indicate that detection of $\mathrm{Cu}(\mathrm{II})$ using NUSIMERs is possible in tissue.

In order to evaluate the possibility to use NUSIMERs in cell experiments, we introduced a fluorescence tag (Atto488) to (2) to obtain the fluorescently labelled NURSIMER (3). We found an average of 5 fluorescence tags per molecule. At first, we have studied the molecular parameters $T_{1}{ }^{-1}$ and $T_{\mathrm{s}}{ }^{-1}$ of (3) in $\mathrm{D}_{2} \mathrm{O}$ and protonated phosphate saline buffer (PBS) to assess the viability for cell experiments (Table 1). We have discovered that (3) does not display a change in $T_{\mathrm{s}}^{-1}$ in protonated buffer with respect to the parent structure (2). In all cases, the equilibration rate $T_{\mathrm{s}}^{-1}$ is shorter than $T_{1}^{-1}$ and therefore represents an excellent starting point not just to filter signals but also to probe molecular dynamics in biological systems. Table 1 summarizes the results.

The observation that a long-lived state can be populated even in aqueous buffered solutions encouraged us to further investigate this behavior in the presence of cells by using

Table 1 Summary of detected longitudinal relaxation rates $\left(T_{1}^{-1}\right)$ and singlet-triplet equilibration rates $\left(T_{\mathrm{s}}{ }^{-1}\right)$ of the macromolecules. Solvents have not been degassed prior to measurements

\begin{tabular}{|c|c|c|c|}
\hline Compound & Solvent & $T_{1}^{-1}\left[\mathrm{~s}^{-1}\right]$ & $T_{\mathrm{s}}^{-1}\left[\mathrm{~s}^{-1}\right]$ \\
\hline G5-PAMAM-GGA-NH ${ }_{2}$ (2) & $\mathrm{D}_{2} \mathrm{O}$ & $2.04 \pm$ & 0.14 \\
\hline G5-PAMAM-GGA-NH ${ }_{2}$ (2) & PBS & $2.85 \pm 0.09$ & 0.06 \\
\hline G5-PAMAM-GGA-Atto488 (3) & $\mathrm{D}_{2} \mathrm{O}$ & $1.4 \pm 0.04$ & $0.32 \pm 0.03$ \\
\hline G5-PAMAM-GGA-Atto488 (3) & PBS & $1.54 \pm 0.09$ & $0.55 \pm 0.01$ \\
\hline
\end{tabular}

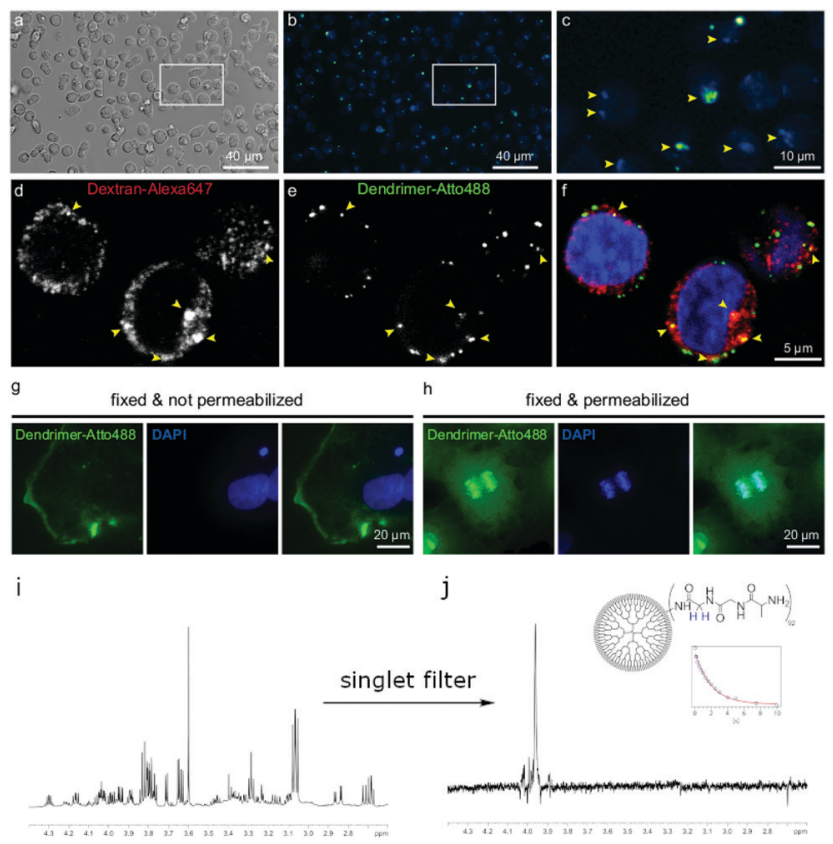

Fig. 6 Different cells and conditions exposed to dendrimers conjugated to Atto488. (a-c) Living human B cell line (RAMOS) exposed to $10 \mu \mathrm{g} \mathrm{mL}^{-1}$ of Atto488 fluorescent dendrimers (3). (a) Transmission light image at low magnification. (b) Epifluorescence image of (a). (c) Magnification from the white rectangle on (a) and (b). Yellow arrowheads show fluorescent dendrimers in the cells. $(d-f)$ Confocal images of RAMOS cells after live co-incubation with (3) and Dextran coupled to Alexa647. Yellow arrowheads indicate co-localization of signals within the optical slide in the confocal $(100 \times$ objective and pinhole set to airy 1$)$. ( $(\mathrm{g}-\mathrm{h})$ Paraformaldehyde fixed COS-7 cells were exposed to (3) with or without the permeabilization of their cellular membranes. (g) (3) stays mostly on the plasma membrane of cells. (h) Permeabilized cells allow the entrance of (3) concentrating in nuclear chromosomes, but also found within the cell cytoplasm/membranes. DAPI stains DNA. (i) Thermal NMR-spectrum of (3) in the presence of RAMOS cells. (j) Singlet filtered spectrum of the same sample. The insert displays the experimentally determined decay in $T_{\mathrm{s}}$.

fluorescence microscopy and the detection of long-lived states. At first, we have successfully tested the uptake of (3) into living human RAMOS B-cell line, in aldehyde-fixed COS-7 fibroblast cells, and in primary rat hippocampal neurons (see ESI $\dagger$ ). ${ }^{65}$ Those first results included the population of a nuclear singlet state in the presence of lysed RAMOS cells in PBS buffered solutions. In another experiment RAMOS B-cells and COS-7 fibroblasts have been co-incubated with (3) as well as Dextran coupled to Alexa647 to determine if (3) are endocytosed in the same fashion as this classical endocytosis marker (Fig. 6). ${ }^{66}$

From the obtained images the internalization of (3) into the cells as well as the localization in the nucleus and the cell membranes could be shown. For subsequent NMR studies, we have focused on B-cells since they are cells growing in suspension. We supplied (3) to a pellet of cells and measured $T_{\mathrm{s}}$. The performed NMR experiments on the B-cells after addition of (3) showed close to no change in $T_{\mathrm{s}}$ which indicates that (3) is stable in the presence of cells. Upon cell lysis, more macromolecules tend to bind to the cell membranes and nuclei. We confirmed this behavior by investigating COS-7 fibroblasts. An 
observed broadening of the NMR-signal of (3) supports the binding hypothesis. Despite the broadening, the signal observation of (3) in the presence of lysed cells was possible using a singlet NMR sequence ${ }^{45}$ with an additional singlet filter. ${ }^{67}$ This filtering experiment allows for direct observation of the glycine protons in (3) while suppressing other proton signals from cell membranes and water with a limit of detection of $6 \mu \mathrm{M}$ concentrations of (3) in a single scan at $900 \mathrm{MHz}$. After extensive washing of the lysed cells, we have still observed (3) due to the possibility of populating singlet states. Additionally, we confirmed the binding by observing an orange coloring of the cells and solid state NMR experiments (see ESI $\dagger$ ). Further experiments on cytosol of B-cells spiked with (3) after lysis and removal of cell membranes showed the stability: we observed no change of the lineshape of (3) and in the measured $T_{\mathrm{s}}$ over a period of several hours. As in the previous experiments, the glycine signal can solely be obtained while suppressing other signals (see Fig. S6, ESI $\dagger$ ).

\section{Conclusion}

In conclusion we observed a unique behaviour of the molecular parameters $T_{1}$ and $T_{\mathrm{s}}$ in NUSIMERs in the presence of different paramagnetic metal ions. Unexpectedly, the presence of Fe(III), Mn(II) and even Gd(III) did not have any effect on neither $T_{1}$ nor $T_{\mathrm{s}}$, which is a behaviour that, to the best of our knowledge, has not been observed so far. Furthermore we were able to observe a unique effect of the presence of $\mathrm{Cu}(\mathrm{II})$ on $T_{1}$ and $T_{\mathrm{s}}$, displaying a sharp decrease in both parameters at low $\mathrm{Cu}$ (II) concentrations followed by an increase at around $150 \mu \mathrm{M} \mathrm{Cu}$ (II). We propose an interaction between the terminal tripeptides of the NUSIMERs and $\mathrm{Cu}(\mathrm{II})$ to be responsible for this observation. The effect of the paramagnetic relaxation competes with a change in the mobility of the tripeptides at the outer sphere of the dendrimers which occurs upon addition of $\mathrm{Cu}(\mathrm{II})$. Based on this observed effect we explored the possibility to use NUSIMERs in an biological environment and evaluate the viability to use NUSIMERs as $\mathrm{Cu}(\mathrm{II})$ probes. We succeeded in attaching a fluorescence tag to the NUSIMERs and observing the uptake in different cells by fluorescence spectroscopy. Using the NUSIMER G5-PAMAM-GGA-Atto488, we were also able to eliminate any background signal using singlet NMR spectroscopy in the presence of cells. This is especially promising, because it opens up the possibility to branch out into $\mathrm{Cu}$ (II) detection in cells or even in vivo, which could in the future lead to a non-invasive technique for early diagnostics of neurodegenerative diseases. This prospect seems especially possible since it has recently be shown, that singlet filtering can be applied in MRI. ${ }^{49}$ Our described experiments could in general serve as an approach to design new molecular probes using nuclear spin singlet states.

\section{Conflicts of interest}

There are no conflicts to declare.

\section{Acknowledgements}

The authors acknowledge generous funding from the Max Planck Society and the Max Planck Institute for Biophysical Chemistry. We thank Prof. M. Bennati for enlightening discussions and access to her EPR instrumentation. K. Overkamp is acknowledged for her support in analyzing the synthesized compounds. We furthermore thank Dr V. Belov and the Chemical Synthesis Facility for a scale-up of compound (2). The authors thank Dr S. Becker and Dr N. Rezaie-Ghaleh for helpful discussions. We thank $H$. Sebesse for support in making the figure. Dr L. Andreas is acknowledged for providing access to the solid state NMR instrumentation. Dr R. Dervisoglu is acknowledged for help in preparing the samples for solid state NMR. We are indebted to Prof. C. Griesinger for access to his facilities. Open Access funding provided by the Max Planck Society.

\section{Notes and references}

1 G. M. Dykes, J. Chem. Technol. Biotechnol., 2001, 76, 903-918.

2 E. Buhleier, W. Wehner and F. Vögtle, Synthesis, 1978, 155-158.

3 M. S. Diallo, S. Christie, P. Swaminathan, J. H. Johnson Jr. and W. A. Goddard, Environ. Sci. Technol., 2005, 39, 1366-1377.

4 V. Balzani, P. Ceroni, S. Gestermann, C. Kauffmann, M. Gorka and F. Vögtle, Chem. Commun., 2000, 853-854.

5 T. D. James, H. Shinmori, M. Takeuchi and S. Shinkai, Chem. Commun., 1996, 705-706.

6 S. B. Garber, J. S. Kingsbury, B. L. Gray and A. H. Hoveyda, J. Am. Chem. Soc., 2000, 122, 8168-8179.

7 J. W. J. Knapen, A. W. van der Made, J. C. de Wilde, P. W. N. M. van Leeuwen, P. Wijkens, D. M. Grove and G. van Koten, Nature, 1994, 372, 659-663.

8 Y. Vida, D. Collado, F. Najera, S. Claros, J. Becerra, J. A. Andrades and E. Perez-Inestrosa, RSC Adv., 2016, 6, 49839-49844.

9 S. P. Kambhampati, M. K. Mishra, P. Mastorakos, Y. Oh, G. A. Lutty and R. M. Kannan, Eur. J. Pharm. Biopharm., 2015, 95, 239-249.

10 F.-H. Liu, C.-Y. Hou, D. Zhang, W.-J. Zhao, Y. Cong, Z.-Y. Duan, Z.-Y. Qiao and H. Wang, Biomater. Sci., 2018, 6, 604-613.

11 A. K. Sharma, L. Gupta, H. Sahu, A. Qayum, S. K. Singh, K. T. Nakhate, Ajazuddin and U. Gupta, Pharm. Res., 2018, $35,9$.

12 S. P. Kuruvilla, G. Tiruchinapally, A. C. Crouch, M. E. H. ElSayed and J. M. Greve, PLoS One, 2017, 12, e0181944.

13 P. Saul, S. Mamone and S. Glöggler, Chem. Sci., 2019, 10, 413-417.

14 M. H. Levitt, Annu. Rev. Phys. Chem., 2012, 63, 89-105.

15 M. Carravetta and M. H. Levitt, J. Am. Chem. Soc., 2004, 126, 6228-6229. 
16 M. Carravetta, O. G. Johannessen and M. H. Levitt, Phys. Rev. Lett., 2004, 92, 153003.

17 M. Carravetta and M. H. Levitt, J. Chem. Phys., 2005, 122, 214505.

18 G. Pileio and M. H. Levitt, J. Chem. Phys., 2009, 130, 214501.

19 G. Pileio, M. Carravetta and M. H. Levitt, Proc. Natl. Acad. Sci. U. S. A., 2010, 107, 17135-17139.

20 G. Pileio, M. Carravetta, E. Hughes and M. H. Levitt, J. Am. Chem. Soc., 2008, 130, 12582-12583.

21 G. Stevanato, J. T. Hill-Cousins, P. Håkansson, S. S. Roy, L. J. Brown, R. C. D. Brown, G. Pileio and M. H. Levitt, Angew. Chem., Int. Ed., 2015, 54, 3740-3743.

22 T. Theis, G. X. Ortiz Jr., A. W. J. Logan, K. E. Claytor, Y. Feng, W. P. Huhn, V. Blum, S. J. Malcolmson, E. Y. Chekmenev, Q. Wang and W. S. Warren, Sci. Adv., 2016, 2, e1501438.

23 Y. Zhang, P. C. Soon, A. Jerschow and J. W. Canary, Angew. Chem., Int. Ed., 2014, 53, 3396-3399.

24 M. C. D. Tayler and M. H. Levitt, Phys. Chem. Chem. Phys., 2011, 13, 5556-5560.

25 G. Pileio, M. Concistrè, M. Carravetta and M. H. Levitt, J. Magn. Reson., 2006, 182, 353-357.

26 T. Theis, Y. Feng, T. Wu and W. S. Warren, J. Chem. Phys., 2014, 140, 014201.

27 S. J. Elliott, L. J. Brown, J.-N. Dumez and M. H. Levitt, Phys. Chem. Chem. Phys., 2016, 18, 17965-17972.

28 R. Buratto, D. Mammoli, E. Chiarparin, G. Williams and G. Bodenhausen, Angew. Chem., Int. Ed., 2014, 53, 11376-11380.

29 R. Buratto, A. Bornet, J. Milani, D. Mammoli, B. Vuichoud, N. Salvi, M. Singh, A. Laguerre, S. Passemard, S. GerberLemaire, S. Jannin and G. Bodenhausen, ChemMedChem, 2014, 9, 2509-2515.

30 Y. Feng, R. M. Davis and W. S. Warren, Nat. Phys., 2012, 8, 831-837.

31 E. Vinogradov and A. K. Grant, J. Magn. Res., 2008, 194, 46-57.

32 W. S. Warren, E. Jenista, R. T. Branca and X. Chen, Science, 2009, 323, 1711-1714.

33 G. Pileio, S. Bowen, C. Laustsen, M. C. D. Wayler, J. T. HillCousins, L. J. Brown, R. C. D. Brown, J. H. Ardenskjær-Larsen and M. H. Levitt, J. Am. Chem. Soc., 2013, 135, 5084-5088.

34 Y. Feng, T. Theis, X. Liang, Q. Wang, P. Zhou and W. S. Warren, J. Am. Chem. Soc., 2013, 135, 9632-9635.

35 Y. Zhang, X. Duan, P. C. Soon, V. Sychrovský, J. W. Canary and A. Jerschow, ChemPhysChem, 2016, 17, 2967-2971.

36 S. Cavadini, J. Dittmer, S. Antonijevic and G. Bodenhausen, J. Am. Chem. Soc., 2005, 127, 15744-15748.

37 P. Ahuja, R. Sarkar, P. R. Vasos and G. Bodenhausen, J. Am. Chem. Soc., 2009, 131, 7498-7499.

38 S. J. DeVience, R. L. Walsworth and M. S. Rosen, NMR Biomed., 2013, 26, 1204-1212.

39 R. Buratto, D. Mammoli, E. Canet and G. Bodenhausen, J. Med. Chem., 2016, 59, 1960-1966.

40 M. C. D. Tayler and M. H. Levitt, Phys. Chem. Chem. Phys., 2011, 13, 9128-9130.

41 R. Sarkar, P. R. Vasos and G. Bodenhausen, J. Am. Chem. Soc., 2007, 129, 328-334.
42 P. Ahuja, R. Sarkar, P. R. Vasos and G. Bodenhausen, J. Chem. Phys., 2007, 127, 134112.

43 N. Salvi, R. Buratto, A. Bornet, S. Ulzega, I. R. Rebollo, A. Angelini, C. Heinis and G. Bodenhausen, J. Am. Chem. Soc., 2012, 134, 11076-11079.

44 A. S. Kiryutin, A. N. Pravdivtsev, A. V. Yurkovskaya, H.-M. Vieth and K. L. Ivanov, J. Phys. Chem., 2016, 120, 11978-11986.

45 S. J. DeVience, R. L. Walsworth and M. S. Rosen, Phys. Rev. Lett., 2013, 111, 173002.

46 C. Stavarache, A. Hanganu, A. Paun, C. Paraschivescu, M. Matache and P. R. Vasos, J. Magn. Res., 2017, 284, 15-19.

47 A. Bornet, P. Ahuja, R. Sarkar, L. Fernandes, S. Hadji, S. Y. Lee, A. Haririnia, D. Fushman, G. Bodenhausen and P. R. Vasos, ChemPhysChem, 2011, 12, 2729-2734.

48 S. Mamone, N. Rezaei-Ghaleh, F. Opazo, C. Griesinger and S. Glöggler, Sci. Adv., 2020, 6, eaaz1955.

49 S. Mamone, A. B. Schmidt, N. Schwaderlapp, T. Lange, D. von Elverfeldt, J. Hennig and S. Glöggler, NMR Biomed., 2021, 34, e4400.

50 M. A. Lovell, J. D. Robertson, W. J. Teesdale, J. L. Campbell and W. R. Markesbery, J. Neurol. Sci., 1998, 158, 47-52.

51 B. S. Gupta, M. Taha and M.-J. Lee, J. Solution Chem., 2013, 42, 2296-2309.

52 X. Wang, X. Cai, J. Hu, N. Shao, F. Wang, Q. Zhang, J. Xiao and Y. Cheng, J. Am. Chem. Soc., 2013, 135, 9805-9810.

53 P. Welch and M. Muthukumar, Macromolecules, 1998, 31, 5892-5897.

54 S. García-Gallego, M. Cangiotti, L. Fiorani, A. Fattori, M. Ángeles Muñoz Fernández, R. Gomez, M. F. Ottaviani and F. J. de la Mata, Dalton Trans., 2013, 42, 5874-5889.

55 D. Lombardo, Biochem. Res. Int., 2014, 837651.

56 M. F. Ottaviani, S. Bossmann, N. J. Turro and D. A. Tomalia, J. Am. Chem. Soc., 1994, 116, 661-671.

57 H. Motschi, Colloids Surf., 1984, 9, 333-347.

58 K. J. de Almeida, Z. Rinkevicius, H. W. Hugosson, A. C. Ferreira and H. Ågren, Chem. Phys., 2007, 332, 176-187.

59 J. V. Folgado, W. Henke, R. Allmann, H. Stratemeier, D. Beltran-Porter, T. Rojo and D. Reinen, Inorg. Chem., 1990, 29, 2035-2042.

60 A. Meyer, G. Schnakenburg, R. Glaum and O. Schiemann, Inorg. Chem., 2015, 54, 8456-8464.

61 S. J. Elliott, C. Bengs, L. J. Brown, J. T. Hill-Cousins, D. J. O'Leary, G. Pileio and M. H. Levitt, J. Chem. Phys., 2019, 150, 064315.

62 J. C. Garcia-Martinez and R. M. Crooks, J. Am. Chem. Soc., 2004, 126, 16170-16178.

63 L. F. Pinto, J. Correa, M. Martin-Pastor, R. Riguera and E. Fernandez-Megia, J. Am. Chem. Soc., 2013, 135, 1972-1977.

64 Z.-J. Chen, G. T. Gillies, W. C. Broaddus, S. S. Prabhu, H. Fillmore, R. M. Mitchell, F. D. Corwin and P. P. Fatouros, J. Neurosurg., 2019, 101, 314-323.

65 M. Maidorn, A. Olichon, S. O. Rizzoli and F. Opazo, MABS, 2019, 11, 305-321.

66 M. A. Gomes de Castro, C. Höbartner and F. Opazo, PLoS One, 2017, 12, e0173050.

67 S. Mamone and S. Glöggler, Phys. Chem. Chem. Phys., 2018, 20, 22463-22467. 